\title{
A Prepaid Smart Metering Scheme Based on WiMAX Prepaid Accounting Model
}

\author{
Reduan H. Khan ${ }^{1}$, Tanzim F. Aditi ${ }^{1}$, Victor Sreeram ${ }^{2}$, Herbert H. C. Iu ${ }^{2}$ \\ ${ }^{1}$ Bangladesh University of Engineering \& Technology (BUET), Dhaka, Bangladesh; ${ }^{2}$ University of Western Australia (UWA), \\ Crawley, Australia. \\ Email: reduan1786@gmail.com
}

Received August $8^{\text {th }} 2010$; revised August $10^{\text {th }} 2010$; accepted August $12^{\text {th }} 2010$.

\begin{abstract}
Prepaid energy meters have been widely adopted by utilities in different countries across the world as an innovative solution to the problem of affordability and consumption management. However, the present smart card based systems have some inherent problems like added cost, low availability and lack of security. In the future Smart Grid paradigm, use of smart meters can completely overhaul these prepaid systems by introducing centralized accounting, monitoring and credit-control functions using state-of-the-art telecommunication technologies like WiMAX. In this paper we propose a prepaid smart metering scheme for Smart Grid application based on centralized authentication and charging using the WiMAX prepaid accounting model. We then discuss its specific application to Demand Response and Roaming of Electrical Vehicles.
\end{abstract}

Keywords: Smart Metering, WiMAX, Smart Grid, Demand Response, Electric Vehicles

\section{Introduction}

Over the last decade, prepaid energy metering has been considered as an effective tool to facilitate affordability and reduce the cost of electricity. Smart card based solid-state energy meters have already been introduced in many parts of the world-especially in the developing countries [1]. Almost all the prepaid energy meters available today are based on smart cards. However, the use of smart cards for utility payment systems has some major drawbacks like added cost, low availability, and risk of complete disconnection due to system failures that outweigh their benefits. In addition, Data storage in a remote meter for a long period poses a number of potential threats like inception, tampering and other fraudulent activities [2].

With the advent of new Smart Grid applications like Demand Response and Plug-in Electric Vehicles, the utility operators need to profoundly change the way prepaid energy systems operate by bringing telecommunications to the core of their activities. Using a smart metering infrastructure with a centralized authentication and charging system, a utility operator can eliminate most of the above mentioned drawbacks and increase the adoption of prepaid energy meters to a large extent. In addition, prepaid energy metering through smart meters provides both the customers and utility operators a wide range of benefits:

1) Enables utility operators to optimize cash flow by charging the user's energy consumption in real-time and giving dynamic offers like discounts and bonuses.

2) Allows customers to purchase energy in convenient ways such as by using credit card online or just sending a SMS from their prepaid phone.

3) Allows the customers to switch from prepaid to postpaid mode seamlessly just by provisioning an unlimited prepaid quota.

4) Enables utility operators to buy electricity back (net metering) easily from renewable and distributed energy sources such as solar, wind, hydroelectric, biomass [3].

5) Allows customers to charge their Plug-in Electric Vehicles (PEVs) while roaming in another utility network.

6) Allows utility operators to execute various load control programs with the aid of Non-Intrusive Load Monitoring (NILM) techniques.

Being the first and only $4 \mathrm{G}$ broadband technologies available today, WiMAX has been emerged as the best candidate to build the next generation Smart Grid Communication network [4]. The open-standard technology platform of WiMAX supports continuous innovation and provides multi-vendor interoperability. In addition, the state-of-the-art authentication, authorization and accounting (AAA) capabilities of WiMAX makes it suit- 
able for real-time smart metering. In this paper we propose a prepaid smart metering scheme for Smart Grid based on centralized authentication and charging using the WiMAX prepaid accounting model [5]. We also present specific applications of the proposed scheme to Demand Response and Roaming of Electrical Vehicles.

The rest of the paper is organized as follows. Section 2 describes the architecture, operation and key features of the WiMAX prepaid accounting model. Section 3 presents a similar architecture for prepaid smart metering, provides a sample usage scenario, and then describes two additional applications-Demand Response and Roaming of PEVs-that the model can support. Finally, Section 4 provides conclusions and directions for future work.

\section{The WiMAX Prepaid Accounting Model}

WiMAX accounting framework is based on RADIUS (Remote Authentication Dial In User Server) protocol [6]. RADIUS protocol generally supports offline (post-paid) accounting. To enable online (prepaid) accounting, a prepaid extension of RADIUS protocol has been released by WiMAX Forum in [7]. Although the model can support a number of pricing schemes, for simplicity, we shall discuss only volume based charging scheme which is relevant to pricing of energy.

\subsection{Architecture}

The architecture of the WiMAX prepaid accounting model is shown in Figure 1. Here, only the network entities relevant to the prepaid charging model will be discussed. A more detail description on the other WiMAX network entities can be found in [8].

A brief description of the WiMAX prepaid accounting entities are given below:

\subsubsection{Network Access Server (NAS)}

The NAS is a generic RADIUS entity. It is the first AAA Client where AAA messages are originated and delivered to. In the prepaid accounting model, the Prepaid Client (PPC) functionality is implemented in the NAS. The PPC interacts with the Authentication, Authorization \& Accounting Server (AAA-S) for dynamic charging information

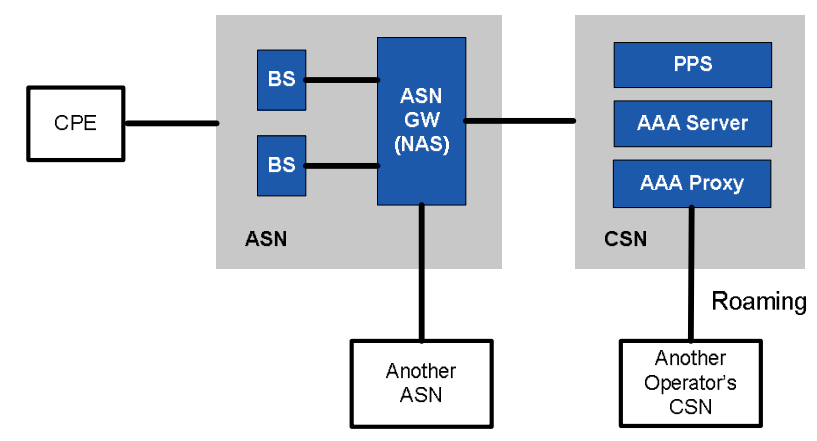

Figure 1. WiMAX prepaid accounting model using RADIUS protocol. The NAS along with PPC is physically implemented in the ASN (Access Service Network) Gateway of WiMAX ASN.

\subsubsection{AAA Server/Proxy}

The AAA-S is typically responsible for authentication of the WiMAX Customer Premises Equipment (CPE). In the prepaid accounting model, the AAA-S communicates with the Prepaid Server (PPS) using RADIUS protocol in order to authorize services to the CPE and perform prepaid accounting and charging.

The AAA proxy transparently routes AAA messages to the destination servers. In roaming scenario, the AAA proxy performs additional interworking functionalities between the home and visited network.

\subsubsection{Prepaid Server (PPS)}

The PPS is located in the WiMAX Connectivity Service Network (CSN). It communicates with the AAA-S \& NAS (PPC) for the purposes of authentication and authorization. In addition, The PPS also performs the following functions:

1) Keeps the subscriber's account balance (Balance manager);

2) Rates access service requests in real-time (Rating Engine);

3) Manages quota for a particular prepaid session (Quota Server).

The PPS checks the subscriber account before authorization. The rating entity in the PPS converts the credit into energy units, called the "quota". This quota is then returned to the requesting PPC.

\subsection{Operations}

The volume based prepaid model is built on an iterative authorization paradigm as the network does not have any prior knowledge about the consumption profile of the customer. In that case, the PPS will reserve a fraction of subscriber's balance into a quota, each time an authorization request is made. The operational procedures of the system are discussed in the subsequent paragraphs.

\subsubsection{Session Initialization}

When a WiMAX CPE attaches to the network, the NAS initiates authentication and authorization procedure by sending a RADIUS Access-Request message to the AAA-S as shown in Figure 2. The message contains standard RADIUS attributes, i.e., the base AVPs (Attribute Value Pairs) along with a special attribute called Prepaid Accounting Capability (PPAC) that indicates the prepaid capabilities of the PPC. The PPAC is a Vendor Specific Attribute (VSA) which is specifically introduced for WiMAX Prepaid Accounting Model.

The PPS checks the subscriber account and authorizes the subscriber. During this procedure, the PPS takes into consideration the capabilities of PPC. Upon successful 


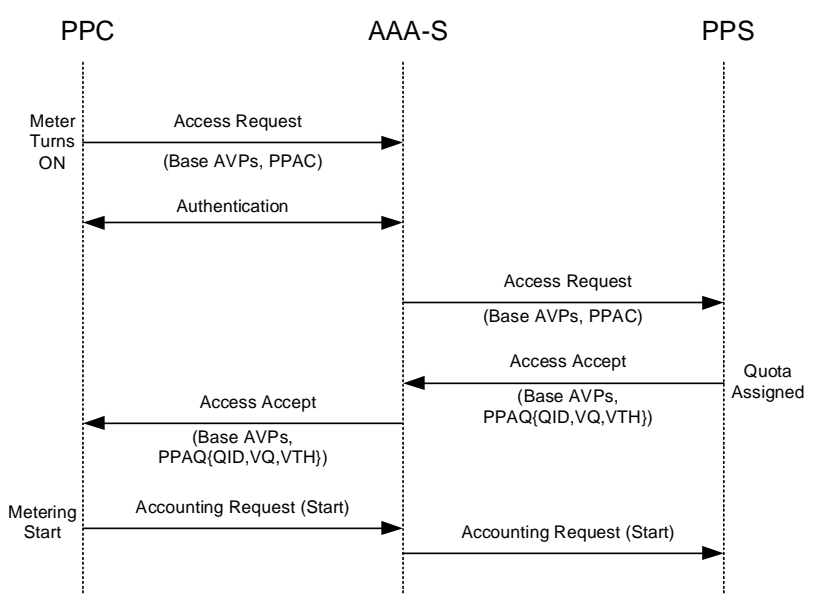

Figure 2. Message flow for prepaid session initialization

authorization, PPS generates an Access-Accept message containing another VSA called PPAQ (Pre-Paid Accounting Quota). The PPAQ attribute includes the following information:

1) Quota ID (QID), which is set by the PPS to a unique value that is used to correlate subsequent quota requests.

2) Volume Quota (VQ), which is set to a value representing a portion of the subscriber's credit.

3) Volume Threshold (VTH) that indicates when the PPC should request additional quota.

4) RADIUS AVP Service Type is set to 6 which means "Authorize-Only".

Upon receiving Access-Accept message from the PPS, the AAA-S appends the usual service attributes and forwards the packets to the PPC. The PPC authorizes the service to the user and starts the metering session. The PPC sends an Accounting-Request (Start) packet to the PPS to indicate the start of the service. Once the Authorize-Only Access-Request message is validated, the AAA-S forwards the Authorize-Only Access-Request to the appropriate PPS.

\subsubsection{Mid Session Negotiation}

When the allocated volume threshold has been reached, the PPC sends an Access-Request message to the AAA-S with PPAQ attributes as shown in Figure 3.

The AAA-S validates the message after verifying the Message-Authenticator field. The PPS locates the prepaid

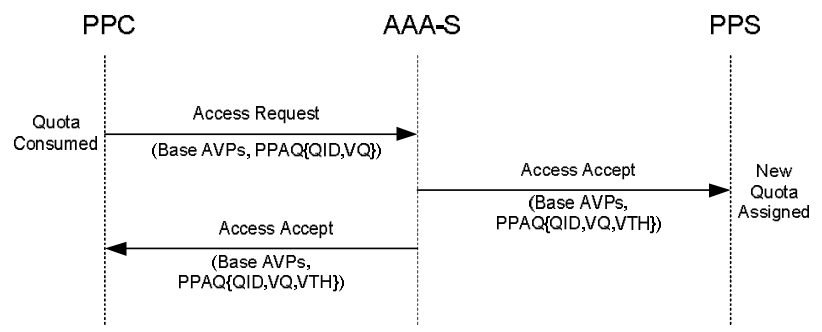

Figure 3. Message flow for mid-session negotiation session state using the QID contained within the PPAQ. The PPS takes the most recently allocated quota and subtracts it from the user balance. If sufficient balance remains, the PPS allocates an additional quota (VQ) and calculates a new threshold value (VTH).

Upon successful re-authorization, the PPS generates an Access-Accept containing the PPAQ attribute. Upon reception of message, the PPC continues to provide service until the new threshold is reached. If the request for additional quota cannot be fulfilled then the PPC lets the subscriber use the remaining quota and terminates the session.

\subsubsection{Session Termination}

The termination phase is initiated when 1) the service is terminated, 2) the subscriber's balance is exhausted, or 3) when the PPC receives an unsolicited Disconnect Message from the PPS. In these cases, the PPC sends an Authorize-Only Access-Request message with a PPAQ and Update-Reason attribute set to either "Client Service Termination" or "Remote Forced Disconnect". Figure 4 shows the RADIUS message flow for session termination operation.

\subsection{Additional Features}

\subsubsection{Tariff Switching}

The model supports tariff switching mechanism based on different time of a day. For example, as shown in Figure 5, peak rate is applied after $6 \mathrm{pm}$. The mechanism requires the PPC to report usage before and after the switch has occurred.

During authentication, the PPC indicates support for tariff switching by setting the appropriate bit in the PPAC. If the PPS needs to inform a tariff switch time, it will send the PTS (Prepaid Tariff Switching) VSA which indicates the point in time when the switch will occur.

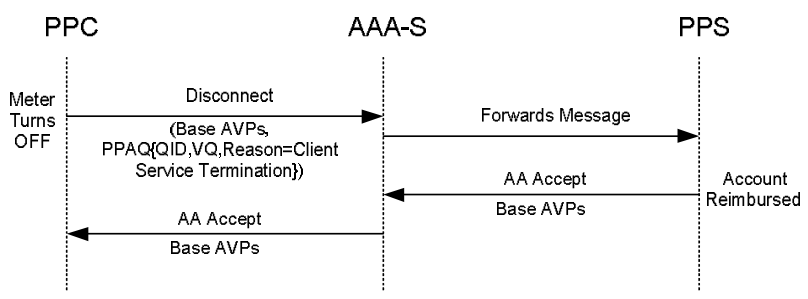

Figure 4. Message flow for session termination

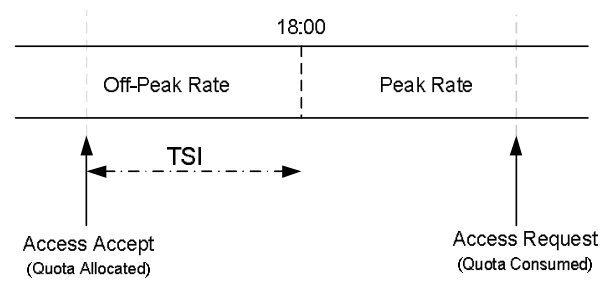

Figure 5. Example of tariff switching 
PTS attribute contains sub-type Tariff Switch Interval (TSI) which indicates number of seconds from current time.

After the interval, the PPC sends another Access-Request message, as a result of either the session being turned off or the volume threshold being reached. The PPC reports how much volume was used in total (in a PPAQ attribute) and how much Volume was Used After the Tariff Switch (VUATS) using the VUATS subtype-attribute in PTS AVP.

\subsubsection{Multiple Concurrent Services}

The model allows differentiation of services within a single session using separate quota for each service and allows for those quotas to be consumed at different rates.

As shown in the Figure 6, a session could be associated with multiple $(\mathrm{N})$ services. Each service is identified by a service identifier (Service-ID). The format of the Service-ID could be expressed as an IP flow using a service context containing Source-IP and Port, Destination-IP and Port and protocol type. Port number can be allocated based on service type. PPS will provide a service ID for each connection inside a session and will allow a separate quota for each service.

\subsubsection{Rating Group}

The model allows a wide range of pricing schemes using "Rating Group" concept that supports complex rating functions efficiently. Rating groups are typically configured at the PPC. As shown in Figure 7, Rating Groups are formed by a group of services from a particular user. However, unlike Figure 6, a single quota is allocated for the entire group.

During the usage of a service that is associated with a Rating Group, the PPC sends the ID of the Rating Group to the PPS. The PPS authorizes the Rating Group by allocating a quota to it. When an additional service that belongs to an already authorized Rating Group is initiated, the PPC meters the service such that it draws from the already allocated quota. Therefore, no RADIUS messages need to be exchanged in this case. This limits the amount of traffic between the PPC and the PPS.

\subsubsection{Support for Roaming}

The model supports roaming scenarios for prepaid charging. In roaming scenarios, the user is always authenticated

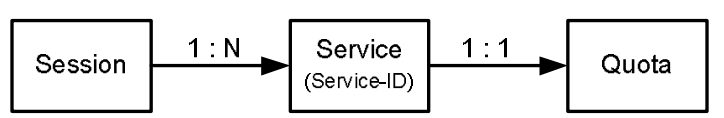

Figure 6. Multiple services within a single session

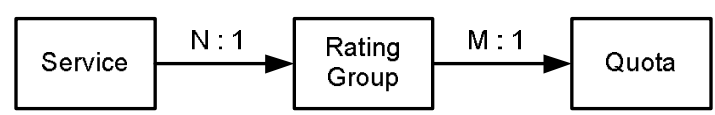

Figure 7. Multiple services with a single quota by its home network. Authorization for the prepaid session and quota replenishing occurs at the home network as well.

\section{The Proposed Smart Metering Scheme}

A similar model, based on the volume based charging scheme of WiMAX prepaid accounting model, can be used for smart-metering. Although there are a lot of differences between a telecom device and a smart-meter, the fundamentals of real-time charging are same. The same iterative charging model can be used, except the fact that, here the subscriber is getting energy service instead of multimedia services.

\subsection{Architectural Model}

Figure 8 shows the architectural model of the proposed scheme. Unlike WiMAX CPE, the metering session is invoked by a WiMAX enabled smart meter in the proposed scheme. Hence, the proposed model is composed of the three logical entities-Smart Meter, ASN and CSNin accordance with the WiMAX prepaid accounting architecture described in Section 2. A brief description of each entity is given below.

\subsubsection{Smart Meter}

The smart meter calculates instantaneous power consumption of the connected load at regular intervals and transmits the data to the nearest WiMAX Base Station (BS). The block diagram in Figure 9 provides a simplified re-presentation of a smart metering circuitry.

As shown in the diagram, the smart meter calculates instantaneous voltage (V) and current (I) of the load using a voltage and current transformer. From V \& I, real power consumption is calculated by a wave-form analysis algorithm using a microcontroller. Then the analogue information is converted into digital values by an ADC

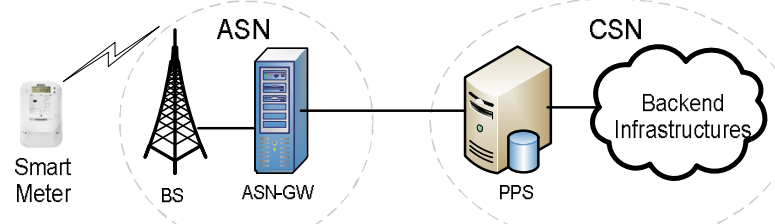

Figure 8. Prepaid charging model for smart metering

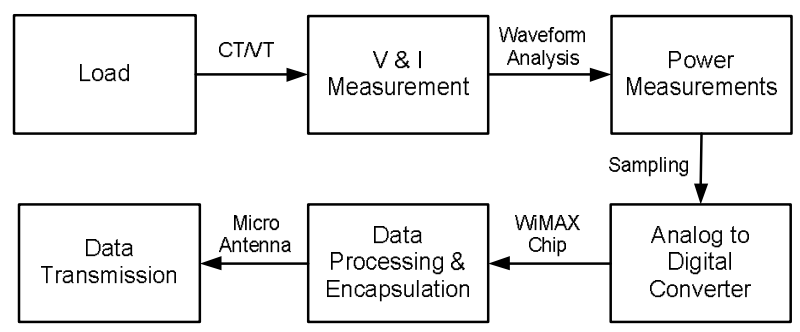

Figure 9. Block diagram of a simple smart metering circuit 
(Analogue to Digital Converter). The digital value is embedded in a metering protocol over the IEEE 802.16 PHY \& MAC PDUs (Protocol Data Unit) by a WiMAX chip and transmitted through its built-in micro-antenna.

To ensure network security, the smart-meter can be periodically authenticated by the AAA server in WiMAX CSN. In addition, the information exchange between Smart Meter and the Core Network can be encrypted by the shared encryption keys received during authentication.

\subsubsection{Access Service Network (ASN)}

The WiMAX BS in the ASN extracts the metering protocol from the IEEE 802.16 PDUs and tunnels them to the ASN-GW over the IP interface using a standard relay protocol. The ASN-GW extracts the power consumption information from the metering protocol and provides it to the PPC.

The PPC in the ASN-GW acts as the charging broker between the Smart Meter \& the Prepaid Server. The PPC must have the capability to analyze the metering information sent by the Smart Meters periodically and convert them into energy units in KWH. The PPC tracks the energy consumption of the meter and requests for a new quota to the PPS once the previous one has been consumed.

\subsubsection{Connectivity Service Network (CSN)}

The main entity in CSN for prepaid smart-metering is the PPS. The PPS updates the account balance based on energy consumption and tariff profile. In addition, it performs additional pricing and load control activities in conjunction with the backend infrastructures. All the communication between PPC and PPS is done via one or more AAA servers/Proxies.

The PPS is connected with several backend infrastructures such as, voucher server for account recharging, billing server for customer invoicing, provisioning system and other application servers for energy management and consumption profiling. A firewall is used to separate the network domains. Figure 10 illustrates the backend connectivity of PPS.

\subsection{Sample Usage Scenario}

To illustrate the functionality of the proposed scheme, we describe a sample usage scenario with the message flows shown in Figure 11:

1) After turning ON, the meter sends a metering request to the PPC using the WiMAX radio network.

2) The PPC opens a session for the meter and sends a RADIUS Access-Request message to the PPS via the AAA.

3) The AAA server authenticates the user, authorizes the service and forwards the Access-Request to the PPS.

4) The PPS verifies the account balance of the user and reserves money equivalent to the initial quota of $5 \mathrm{KWH}$.

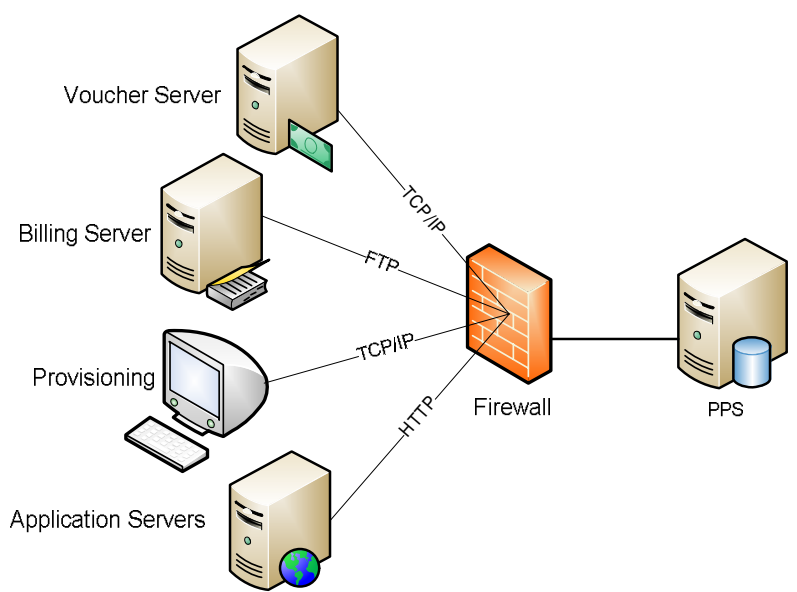

Figure 10. Connectivity of PPS with the backend infrastructures

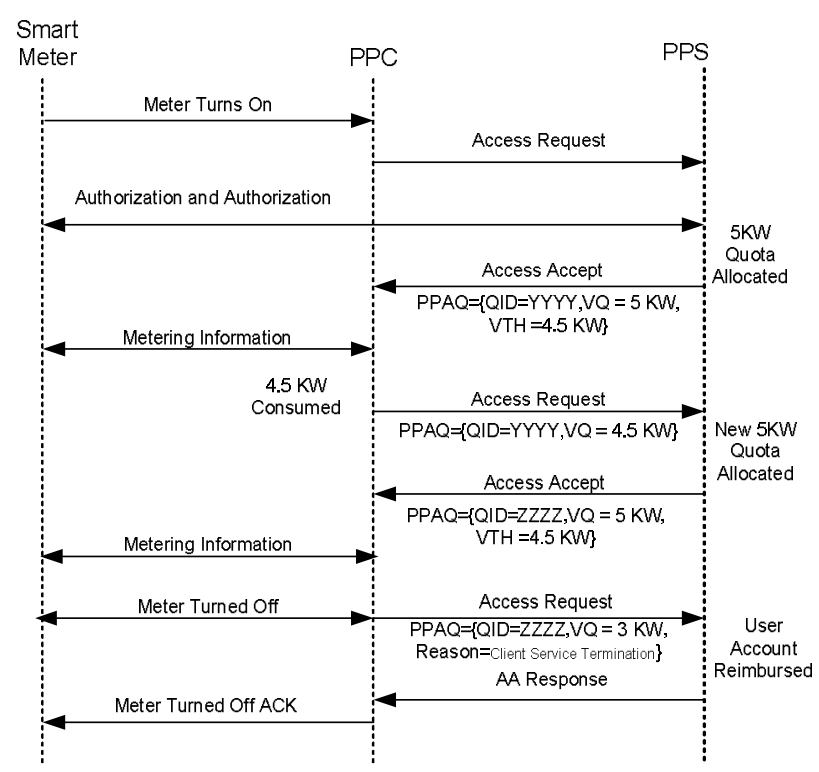

Figure 11. Message flow for the proposed charging model

Then it sends an Access-Accept message to the PPS with a Volume-Quota (VQ) of $5 \mathrm{KWH}$ and Volume-Threshold (VTH) indication of $4.5 \mathrm{KWH}$. It further associates the Quota Identifier (QID) $=$ YYYY to this quota reservation.

5) Upon reception of message, the PPC continues the user session and meters the energy consumption periodically.

6) When the allowed 4.5 KWH energy has been consumed by the user, the PPC generates an Access-Request that contains the amount of consumed quota, and the request for replenishment.

7) Upon reception of message, the PPS identifies the user from the QID and PPS subtracts the reserved money from the account. Then it sends an Access Accept message to the PPS with a new VQ of $5 \mathrm{KWH}, \mathrm{VTH}$ of 4.5 
KWH and a new QID as ZZZZ.

8) Upon reception of message, the PPC updates its records and continues provisioning energy access to the user. The procedure runs iteratively until the prepaid account balance of user has been used up or the energy consumption has been stopped.

9) In case, the meter is turned off or a re-authentication procedure is initiated, the PPC sends an Access-Request message with the reason "Client Service Termination" and specifies the amount of energy consumed from the allocated quota. The PPS subtracts the portion of the reserved money equivalent to consumed energy and reimburses the balance.

\subsection{Applications}

In addition to the real-time prepaid charging, the proposed scheme can support the following two major smart grid applications using the additional features of WiMAX prepaid accounting model described in Section 2.

\subsubsection{Demand Response}

Demand response enables the utility operator to optimally balance between power generation and consumption either by offering dynamic pricing or by implementing various load control programs [9]. Using the proposed scheme that supports tariff switching, charging of multiple services in a single session, rating groups, utility operators can perform various demand response programs effectively and efficiently with minimum additional costs.

For example, during peak hours, the utility company can charge a premium rate for the non-essential appliances such as electric heater, air-conditioner and washing machine using the tariff switching functionality. Furthermore, the rating group feature of the proposed scheme allows the utility operator to offer flexible pricing schemes to its customers and thus maximize their profit. For example, a utility operator may wish to rate energy such that the first $\mathrm{N} \mathrm{KWHs}$ are free, then the next M $\mathrm{KWHs}$ are rated at $\$ 5$ per $\mathrm{KWH}$ and volume above $(\mathrm{N}+$ M) KWH be rated at $\$ 6$ per KWH.

Demand Response using automatic load control can also be performed using an "Unsolicited Session Termination" operation on a particular group of loads and thus reduce the load from the grid. The differentiation of loads can be performed by using non-intrusive load monitoring techniques as described in [10]. In this case, as shown in Figure 12, the PPS should dynamically interact with a SCADA server located in the WiMAX CSN which will manage and implement the load-control programs via the WiMAX ASN.

\subsubsection{Roaming of PEVs}

PEVs are regular hybrid vehicles that have a large highcapacity battery bank. The batteries on these vehicles can take power from the grid during off-peak hours to charge the batteries and provide power back to the grid during peak hours. Thus they can play a key role in balancing power generation and consumption of the grid [11]. Since the PEVs are highly mobile, integrating them into the Smart Grid can be a major challenge.

The proposed scheme enables utility operators to allow flexible charging and de-charging of PEVs in any utility network using just an on-board smart meter. Although PEVs are mobile, the charging stations are static. Therefore, no mobile communication network is needed. Figure 13 shows the roaming scenario of a PEV with an on-board WiMAX smart meter.

The charging station is located at the visited WiMAX network. The smart meter in the PEV authenticates itself and sends metering information to the AAA and Prepaid Server of its home network. The communication between home and visited network is done via the AAA proxy servers located at the edge of each network. Here, the visited network merely provides network access to the PEV.

\section{Conclusions}

Prepaid charging of energy has the potential to provide both customers and utility operators a wide range of benefits including consumption regulation, load management and integration of distributed energy resources. In this paper, we have provided a detail study of WiMAX prepaid accounting model along with its key additional features. This study is then used to present a similar prepaid charging scheme for smart metering to be used in Smart Grid. Specific applications of the proposed scheme

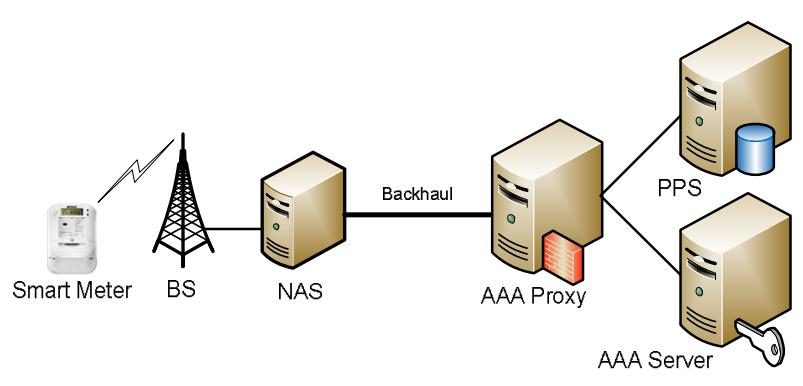

Figure 12. Automatic load control using the prepaid model

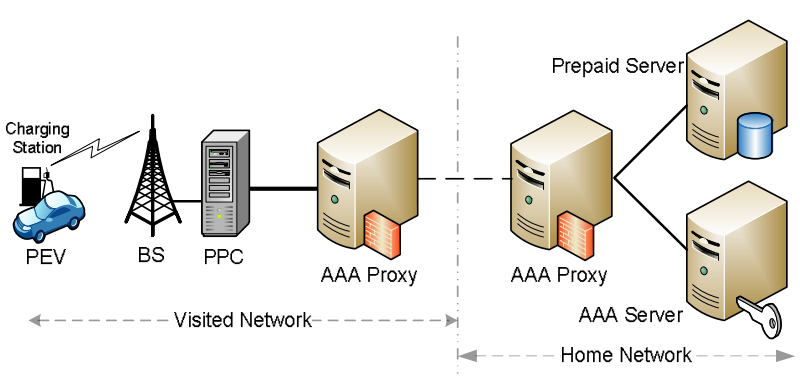

Figure 13. Prepaid Roaming of Electric Vehicles 
to Demand Response and Roaming of Electrical Vehicles are also discussed.

The proposed scheme makes use of existing WiMAX core infrastructure as much as possible while providing secure charging using the state-of-the-art security capabilities of WiMAX network. Since the scheme is based on a telecom framework, it requires a separate protocol to transfer the metering information from smart meters to the WiMAX core network. In addition, the scheme needs specific guidelines for data storage, session maintenance, authentication, quota allocation, and handling of outage and quality related events.

The continuation of the research will include development of necessary protocols and guidelines for supporting various metering, control, and pricing applications on the proposed scheme. An experimental examination on the performance of the proposed scheme is also worth further investigation.

\section{REFERENCES}

[1] A. A. Casarin and L. Nicollier, "Prepaid Meters in Electricity. A Cost-Benefit Analysis," IAE Business School, Austral University, March 2008. http://www.sandiego.edu

[2] P. McDaniel and S. W. Smith, "Security and Privacy Challenges in the Smart Grid," IEEE Computer and Reliability Societies, Los Alamitos, 2009

[3] Whitepaper, "Electricity Smart Metering: Business Drivers," Atos Origin, November 2009. http://www.fr.atosorigin.com/

[4] Whitepaper, "WiMAX Applications for Utilities," WiMAX Forum, October 2008. http://www.senza-fili.com/

[5] "WiMAX End-to-End Network Systems ArchitectureStage 2: Architecture Tenets, Reference Model and Reference Points," WiMAX Forum, November 2005.

[6] C. Rigney, A. Rubens, W. Simpson and S. Willens, "Remote Authentication Dial In User Server (RADIUS)," RFC 2856, Internet Engineering Task Force, June 2000.

[7] "WiMAX End-to-End Network Systems ArchitectureStage 3: Detailed Protocols \& Procedures, Annex: Prepaid Accounting," WiMAX Forum, February 2009.

[8] Whitepaper, "Mobile WiMAX-Part I: A Technical Overview and Performance Evaluation," WiMAX Forum, March, 2006. http://www.wintegra.com/

[9] S. Braithwait and K. Eakin, "The Role of Demand Response in Electric Power Market," Edison Electric Institute, October 2002. http://www.smartgridnews.com

[10] C. Laughman, K. Lee and R. Cox, "Power Signature Analysis," IEEE Power and Energy Magazine, Vol. 1, No. 2, 2003, pp. 56-63.

[11] A. Brooks, T. Gage and A. C. Propulsion, "Integration of Electric Drive Vehicles with the Electric Power Grid-A New Value Stream," 18th International Electric Vehicle Symposium and Exhibition, Berlin, October 2001. http://www.smartgridnews.com 